\title{
Appareil
}

$20 \mid 2018$

Arts contemporains et patrimoine

\section{Idée esthétique : prise de site}

Jean-Louis Déotte

\section{(2) OpenEdition}

Journals

Édition électronique

URL : http://journals.openedition.org/appareil/2922

DOI : 10.4000/appareil.2922

ISSN : 2101-0714

Éditeur

MSH Paris Nord

Référence électronique

Jean-Louis Déotte, «Idée esthétique : prise de site », Appareil [En ligne], 20 | 2018, mis en ligne le 26 octobre 2018, consulté le 30 juillet 2020. URL : http://journals.openedition.org/appareil/2922 ; DOI https://doi.org/10.4000/appareil.2922

Ce document a été généré automatiquement le 30 juillet 2020.

\section{(c) (i) (2) $\Theta$}

Appareil est mis à disposition selon les termes de la Licence Creative Commons Attribution - Pas d'Utilisation Commerciale - Pas de Modification 4.0 International. 


\title{
Idée esthétique : prise de site
}

\author{
Jean-Louis Déotte
}

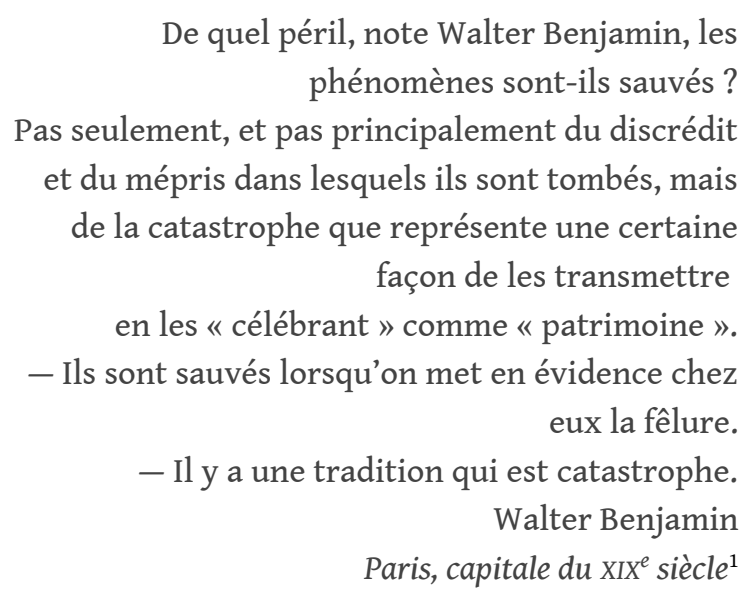

1 Je propose de continuer notre réflexion sur la notion de "prise de site » entendue comme développement critique (au sens où l'entendent les Romantiques d'Iéna), par des installations contemporaines, dans un édifice fixé par la patrimonialisation, c'est-à-dire par la mise en exposition.

2 C'est évidemment le cas de l'Hôtel-Dieu de Tonnerre qui a, de même qu'une œuvre d'art dans un musée, un statut que la notion de suspension caractérise bien. C'est devenu un « suspens » même s'il s'agit d'un édifice. C'est un texte dont le contexte a disparu. Là où se trouvait autrefois une cité hospitalière réunissant toutes les fonctions répondant au programme établi par Marguerite de Bourgogne à la fin du XIII ${ }^{\mathrm{e}}$ siècle, sans oublier le château de la comtesse relié à la nef par une passerelle, ne subsiste qu'un bâtiment, le Pressoir, isolé le long de la rivière. Tout ce qui se trouvait autour a été démoli, y compris désormais les grilles séparant cette cité hospitalière de la ville de Tonnerre.

Un édifice patrimonial est nécessairement un «bel " édifice, à la monumentalité glorieuse, débarrassée des niches adventices où toute une population avait réussi à s'installer. Le processus de patrimonialisation est à rapprocher de la taille d'un bloc de marbre classique ou de la taille par un diamantaire. Ce processus est par ailleurs indissociable de l'émergence d'un appareil esthétique qui lui est contemporain: le 
musée. C'est le même Quatremère de Quincy qui détachera la Fontaine des Innocents, à Paris, de l'ancienne église des Saints-Innocents - contribuant ainsi à l'isoler sur une place - et qui rédigera le portrait le plus féroce qui soit de l'institution muséale ${ }^{2}$. L'illumination externe puis interne d'un édifice renforce cette esthétisation comparable à une mise sous vitrine. Parallèlement à ce surcroît d'exposition, la valeur de culte du monument tend à disparaitre. On hérite ainsi de monuments sans destination effective, vidés de toute substance onto-théologique. C'est à ce prix que la valeur esthétique peut être isolée et peut dominer. On n'ignore pas que cette double esthétisation est inséparable de discours, de décrets et de la création de corps de fonctionnaires chargés de les mettre en œuvre (Mérimée sous le Second Empire, etc.); et plus précisément, en ce qui concerne les monuments historiques, de la littérature. De là résulte l'aspect fantasmagorique des restaurations d'un Viollet-le-Duc.

Mais à la différence d'un musée qui est au service des suspens qu'il abrite (pas de musée sans collections d'œuvres), les installations artistiques d'un site du patrimoine en font la critique, c'est-à-dire qu'idéalement, elles en développent telle ou telle possibilité architecturale ou esthétique en général. Voilà le sens de la commande que nous passons, à Tonnerre, aux artistes : amplifier telle ou telle potentialité de l'édifice; ou proposer telle ou telle utopie restée en creux, nous attendant dans le passé, ainsi que l'écrit Benjamin dans ses thèses sur le concept d'histoire :

N'est-ce pas autour de nous-mêmes que plane un peu de l'air respiré jadis par les défunts? N'est-ce pas la voix de nos amis que hante parfois un écho des voix de ceux qui nous ont précédés sur terre ? Et la beauté des femmes d'un autre âge, estelle sans ressembler à celle de nos amies ? C'est donc à nous de nous rendre compte que le passé réclame une rédemption dont peut-être une toute infime partie se trouve être placée en notre pouvoir. Il y a un rendez-vous mystérieux entre les générations défuntes et celle dont nous faisons nous-mêmes partie. [...] Car il nous est dévolu à nous comme à chaque équipe humaine qui nous précéda, une parcelle du pouvoir messianique ${ }^{3}$.

5 «Être attendu sur terre [...] posséder une parcelle de pouvoir messianique, réclamée par le passé ", c'est cette nécessité qui fait défaut aux œuvres des arts contemporains, qui ne font que répondre aux exigences de la nouveauté. C'est ce partage essentiel qui légitime la confrontation avec un édifice patrimonial. On pourra se servir autant qu'on le voudra de ces édifices pour y installer des œuvres in situ, on ne répondra pas pour autant à cette nécessité.

6 Il est évident que n'importe quel suspens muséal peut revendiquer ce "pouvoir messianique ", mais c'est en propre ce à quoi nous engage un édifice appartenant au patrimoine s'il échappe au statut de "bien culturel », ce qui est très exactement le statut des Hospices de Beaune par exemple, dans la mesure où toute fêlure a été masquée par un exercice de fiction très réussi qui relève de la fantasmagorie. Il n'y a pas de fêlure dans une fantasmagorie : le fantasme absorbe toute césure.

7 On peut ramener à la vie ces Hospices en remontant à la source, à leur "préhistoire ", en regardant les dessins d'aménagement de l'Hôtel-Dieu de Tonnerre par Viollet-le-Duc comme des utopies architecturales, autrement dit artistiques et non pas archéologiques. Car, au XIX ${ }^{\mathrm{e}}$ siècle, ce cher sauveteur des ruines gothiques avait été pris au pied de la lettre. Dès lors, Tonnerre était devenu le modèle des Hospices de Beaune : une pure fantaisie néo-romantique.

8 Par «critique », il ne faut rien entendre de négatif. Pour les Romantiques, comme les frères Schlegel ou encore Novalis, il s'agit un enchaînement nécessaire qui consiste en 
un enrichissement ${ }^{4}$. Pour eux, une œuvre peut ainsi être la critique d'une autre, un art, celle d'un autre, le plus abouti étant la poésie lorsqu'elle vise l'art comme absolu. Cette amplification continue a une raison: c'est parce qu'une œuvre est un «fragment d'avenir » qu'elle suscite la reprise.

9 Les installations de Caroline Coppey (2014), Didier Guth (2015) et Germain Roesz (2016) furent à cet égard de parfaites réussites, parce que ces artistes comprirent que leur regard critique était attendu. Plus que de rendre davantage visible un édifice qui est déjà largement surexposé, ce qui serait le cas avec des œuvres in situ, ces installations éphémères, générées sur place, ne durèrent qu'un instant, comme dans une rêverie. La dernière fut peut-être la plus complexe : Germain Roesz s'était servi d'une matrice en bois reprenant la sinusoïdale de la trace gravée sur le sol par la méridienne du XVIII ${ }^{\mathrm{e}}$ siècle. D'où le titre de son installation : L'Entaille de la lumière. Dès lors, l'amplitude de la courbe était telle que le spectateur la mettait inévitablement en rapport avec la courbe en vaisseau, légèrement surbaissée, de la voûte. Cette interprétation artistique proposait une thèse technologique : cette voûte était-elle techniquement parfaite au sens où l'entend Simondon (auto-soutènement idéal de la voûte " en chaînette ") ? Les immenses peintures verticales de Roesz témoignaient de son activité en atelier, des arcs déployés à partir de sa matrice, mais aussi du déplacement du soleil sur le sol de la halle suivant les mois de l'année. Pour les Romantiques, un fragment d'avenir peut aussi être une ruine, la mémoire de l'activité solaire annuelle.

10 Les installations prises de site seraient au patrimoine ce que les suspens sont au musée, mais en inversant le sens des rapports. La notion d'individuation, chère à Simondon, peut s'avérer utile en venant constituer le cadre commun de ces rapports de rapports : le musée individue les œuvres qui ont été de culte en les transformant en suspens. Ce faisant, le musée annule leur capacité à générer une communauté de "destin », mais révèle leur valeur esthétique. La Joconde, la Victoire de Samothrace, la Vénus de Milo ont ainsi individué le Louvre comme le feraient des œuvres in situ. Au contraire, l'édifice patrimonial qui n'a plus qu'une valeur esthétique et historique est individué par des installations éphémères qui n'ont qu'un intérêt esthétique secondaire (comme l'outil visuel chez Buren, le Pointillé blanc de Didier Guth dans l'exposition «... et les marguerites»). La nécessité de ces installations prises de site s'impose dans le vide intérieur de ces architectures privées de destination. Dès lors, les artistes qui sont intervenus ont eu à affronter cette fêlure. La tentation était grande d'utiliser des pièces déjà existantes et de procéder à des accumulations hétéroclites qui pouvaient passer pour poétiques ${ }^{5}$, néo-surréalistes. Nous avons très tôt refusé cette option qui aurait transformé le site en lieu pour l'art contemporain. Ce lieu, nous l'avons ouvert à quelques pas, dans l'ancienne pharmacie du centre hospitalier. C'est devenu La Pharmacie, qui a accueilli sa première exposition à l'été 2017 : Prendre soin ${ }^{6}$. On peut caractériser cette première exposition, suivant Benjamin, comme une exposition de déniaisement, portant sur l'usage contemporain des médicaments, sur leur falsification, le trafic mondial, l'addiction ${ }^{7} .$.

11 Cette halle médiévale n'est pas un habitat; elle est, par sa démesure, un défi. Une impossibilité. Comme l'a fort bien montré Jacques Boulet, on n'en voit pas la vocation. C'est une énigme, ce n'est pas un Hôtel-Dieu inaugurant la série de Tournus, Louhans, Cluny, Beaune, etc. L'espace y est sublimé, d'où la métaphore chimique de la " prise » d'espace. L'artiste ne peut se conduire en conquérant : son installation peut « prendre " ou pas. Dans le cas d'une installation in situ, il y a un face-à-face entre le spectateur et le 
site. Dans le cas d'une prise de site, le spectateur se trouve inclus, comme on peut l'être dans la salle des Nymphéas au musée de l'Orangerie, à Paris. Or, puisque Monet était le contemporain du cinématographe $e^{8}$, on sera sensible aux édifices patrimoniaux que viendrait sauver le cinématographe ${ }^{9}$.

On ne peut pas ne pas être dépris par ce site. Les œuvres in situ sont encore un avatar de l'écriture projective; les installations prises de site, quant à elles, relèvent de l'inclusion, de l'absorbement numérique ${ }^{10}$.

Pour rapporter les propos de Jacques Boulet, lors d'une de nos correspondances :

Ce qui me gêne dans l'expression in situ, c'est que les œuvres qui s'en réclament ou qui y sont rapportées par une critique, présupposent l'existence d'un site et d'un dedans limité ou illimité, et partant d'un placement.

Il précise ensuite :

Ce que je note dans les œuvres de Coppey, de Guth et de Roesz, c'est qu'au-delà de toutes les inépuisables résonances allusives, analogiques, mimétiques avec l'espace qualifié de la nef, toutes ont engagé une déprise du site, un au-delà et un ailleurs. Déprise du site, comme un « ici » à partir d'où commence l'œuvre, l'art... le poème. [...] Mon expérience des œuvres d'art [prise de site], qui me porte souvent jusqu'à l'excitation plaisante, déplaisante ou autre, d'une "généalogie de l'unique " [l'individuation] m'incite à une toute autre interprétation des œuvres, ou culture de mon propre rapport à l'art. J'y éprouve la banalité de l'instauration non pas d'un site, mais d'un espace avec ses déplacements, sa réversibilité, sa temporalité, l'étrange sensation de mon inclusion dans une œuvre, dans un espace dont j'ai la liberté d'en fixer les limites réelles ou imaginées, un espace évidemment qualifié historiquement, socialement, politiquement, etc., qui n'est plus ce lieu, mais un hors lieu de par l'œuvre.

15 Alors, pourquoi ne pas considérer ce lieu hors lieu rendu possible par l'œuvre, ce lieu imaginaire ? Ou fictif ? C'est ce lieu que nous avons essayé d'ouvrir dans les trois textes consacrés à ces artistes. Car, là où un historien d'art classique comme Panofsky étagera trois niveaux interprétatifs (description matérielle, iconographie, iconologie), réduisant l'œuvre à un énoncé, nos "preneurs de site " alimenteront des fictions théoriques qui n'ont de légitimité que parce qu'elles prolongent des rapports de rapports. Nous avons ainsi pu mettre en rapport Le Grand Voile de Caroline Coppey avec les structures géométriques et décoratives des muqarnas de l'art islamique ; le pointillé blanc de Didier Guth avec l'appareillage de la mémoire selon Freud; les arcs-arches de Germain Roesz avec les différentes courbes de la nef.

Ces fictions artistiques ont répondu au cadre protocolaire, engageant une réception esthétique que va constater la prise de site. Que cela tienne ou pas, telle est la qualification du plaisir esthétique. Ce n'est qu'à ce prix, selon Benjamin, qu'il y aura un sauvetage possible du patrimoine du passé. Sinon, c'est la valeur de nouveauté qui l'emportera... cette détermination de la mode.

17 Un élément d'une prise de site peut devenir une pièce de musée, un suspens, à la manière du Grand Voile de Caroline Coppey, mais ce sera à la suite d'un déplacement qui en changera la fonction. L'œuvre passe alors d'un site ayant eu une histoire complexe à un lieu quelconque dont l'histoire importe peu, où les seules caractéristiques sont quantitatives (white box). Or l'enjeu politique, c'est le sauvetage du passé, sans lequel les arts contemporains seront absorbés par le marché.

18 La doxa contemporaine a usé et abusé du concept de "concept ». C'est devenu l'idée pour le marketing ou pour la communication. Redonnons au concept son appartenance 
à la connaissance : pas de connaissance sans concept empirique ; pas de réalité de cette connaissance sans référent (sensible) pour le concept, c'est-à-dire sans existence d'un objet pour lequel il y a un concept. On peut avoir un concept de Dieu, mais sans la possibilité de le désigner en fonction de déictiques (ici, maintenant, devant, derrière, hier, demain, je, tu, etc.), il n'y a pas de réalité de Dieu. Les « preuves de l'existence de Dieu » peuvent être rationnelles, elles n'attestent en rien sa réalité. La foi peut être rationnelle, la seule rationalité ne suffit pas pour conférer l'existence, qui demande un constat. A contrario, l'analogie de la ressemblance sensible, dite mimétique, n'apporte aucune connaissance. Dès lors, entre la métaphore sensible et l'analogie quantitative, mathématique, il y a une voie moyenne : on peut suggérer l'existence d'un objet par une mise en rapport de rapports. Exemple canonique $: a / b=c / d$. Connaissant $a, b$ et $c$ on peut en déduire $d$, supposant que les entités mises en relation appartiennent au même domaine de réalité. Benjamin, qui cherchait des ressemblances non mimétiques, scripturaires, a souvent procédé de cette manière, tout en ne précisant pas explicitement quel était le domaine d'appartenance commune. On connaît cette analogie: "le peintre est au caméraman ce que le chaman est au chirurgien ${ }^{11}$ ». Le peintre, le chaman et le chirurgien étant des entités connues, quelle est la nature du cameraman?

On peut suggérer ce développement :

\begin{tabular}{l} 
peintre \\
\hdashline chaman \\
cameraman
\end{tabular}$=\begin{gathered}\text { une fois pour toutes } \\
\text { chirurgien }\end{gathered} \quad \begin{aligned} & \text { une fois n'est rien } \\
& \text { unra }\end{aligned}=\begin{aligned} & \text { ici et maintenant } \\
& \text { trace }\end{aligned}$

19 Il y a donc un faisceau de points de vue possibles : selon la temporalité (l'événement); selon la présence/absence (la trace s'opposant à l'aura ${ }^{12}$ ); selon l'époque de la technique: artisanale/industrielle (soit l'unicité de l'œuvre/soit l'essentielle reproductibilité); etc. Il y en d'autres: la perception (soit contemplative/soit «distraite ») ; l'extériorité de la narration mythique/la pénétration du corps par des instruments ; ou encore le don et le sacrifice de soi ; le jeu...

Dès lors, on peut conclure comme suit : le caméraman, technicien de la seconde époque, pénètre avec sa caméra le corps du collectif. Il peut toujours recommencer la prise de vue, car l'acteur n'est pas un comédien (absence du public ici et maintenant); il n'y a plus d'unicité de l'œuvre, mais des copies toujours modifiables. L'esthétique du film procède par discontinuités, elle provoque des chocs plus sensoriels ou physiologiques qu'un plaisir purement esthétique.

On ne peut donc pas se contenter des analogies qualitatives, il faut préciser ce qu'est le domaine commun des rapports comparés, leur plan d'immanence (Deleuze). En ce qui concerne notre questionnement, ce doit-être une idée esthétique au sens de Kant, proche de l'idée spéculative, laquelle fait défaut à bien des installations in situ.

Résumons : la prise de site dans un édifice comme l'Hôtel-Dieu suppose au préalable une mise en rapport de rapports. L'enjeu est celui d'une ressemblance non sensible, non mimétique, mais cela n'est possible et ne trouve sa nécessité que par la mise en œuvre d'une idée esthético-spéculative. Cette articulation entre un édifice et sa prise de site est probablement la seule voie pour échapper au marché de l'art, car l'amateur, qui est le plus souvent un spéculateur, ne peut alors se satisfaire de produits dérivés. Si le mobile de la spéculation sur l'art est la distinction (Baudrillard, Bourdieu), la sphère artistique disparaît au profit de la compétition sociale. 

possible, c'est celui de la poésie (le romantisme hégélien). A contrario, c'est l'idée qui ouvre le champ de la production artistique à l'époque de la domination du marché et donc de la philosophie esthétique.

le cas d'Entaille de la lumière, de Germain Roesz (2016), on peut montrer la nécessité d'une telle production picturale. La courbe de la voûte est à la sinusoïdale de la trace portée par le soleil sur le sol de la méridienne (1786) ce qu'est la courbe de l'arc en bois (qui servira de matrice) aux traces colorées sur les différentes pièces de tissu suspendues aux poutres, comme sur les reprises dans les triangles placés sur les murs. Les rapports sont de même nature, ce qui permet l'analogie qualitative. Ce sont des rapports d'individuation (Simondon) : la sinusoïdale est une individuation du demicercle (de la voûte), comme le sont, pour la matrice de l'artiste, les différentes traces peintes sur différents supports (tissu, bois, etc.). Le site est ainsi doublement pris en charge : répétition de la voûte, inscription annuelle du parcours du soleil grâce au gnomon gravé sur le sol. On pourrait en dire autant des différents triangles constitués par la charpente, en particulier de ceux accessibles au-dessus de la voûte: ils constituent le second élément de la matrice en bois utilisée par Roesz. Mais alors, l'individuation va de ces triangles quelconques aux triangles parfaits de la poutraison. L'idée d'individuation est bien l'idée esthétique qui permet de rendre compte de cette prise de site.

D'autres rapports sont possibles, si l'on met en œuvre un imaginaire qui ne dédaigne pas les proportions, les appareils cosmétiques (Caroline Coppey) ou esthétiques (Didier Guth). Découvrant l'immense volume vide de la halle, Viollet-le-Duc s'ingénia à restructurer l'ensemble en s'appuyant sur certaines archives. Constatant l'existence régulière de deux percements sous chaque haute fenêtre gothique, il en déduisit l'existence ancienne d'une galerie faisant le tour complet de la nef. Elle aurait permis l'éclairage de la salle et la surveillance des malades placés dans des lits perpendiculaires aux parois (Voir les dessins de Viollet-le-Duc). Or, ce que l'on sait de l'existence de salles d'apparat de moindre dimension, comme la salle de l'Échiquier ${ }^{13}$, où le duc de Normandie avait l'habitude de réunir les siens, permet d'imaginer une salle à deux niveaux : un niveau bas destiné à la réception des pèlerins, des étrangers, des pauvres, des affamés et des assoiffés, au stockage des vivres, au logement des sœurs; et une salle haute reposant sur des entraits constituant un sol soutenu par des étais. Dans le cas de Caen, cette salle basse est aveugle comme elle aurait pu l'être à Tonnerre. Dès lors, l'idée (théologique) de la salle haute serait celle de la lumière dans laquelle baigneraient les malades, mais aussi l'entourage de Marguerite de Bourgogne. La tribune ouvrant sur le décor classique de la salle Courtanvaux (actuel musée de l'Hôpital) aurait été de niveau avec le sol de la salle d'apparat; le Cellier serait, quant à lui, au niveau de la salle basse ${ }^{14}$. L'existence d'un fossé entourant l'édifice, sans usage de nos jours, permettrait de renforcer cette hypothèse. Autant de rapports, à partir de la comparaison entre Caen et Tonnerre ${ }^{15}$, qui permettent de développer d'autres idées esthétiques. Et donc d'autres installations artistiques...

On dira d'une œuvre qu'elle est "prise de site» si elle n'entretient aucun lien mimétique ou métaphorique avec le site, mais se trouve en rapport avec l'une de ses articulations majeures.

Si l'on résume les caractéristiques de l'Hôtel-Dieu :

- une salle basilicale à la voûte surbaissée qui est un chef-d'œuvre de la charpente gothique ;

Appareil, $20 \mid 2018$ 
- une salle surdimensionnée à la recherche d'une masse de volume. La charpente gothique permettant de franchir de grandes sections ;

- la volonté d'unifier l'espace comme à Bourges : une seule nef alors qu'il y en a cinq à NotreDame, pour unifier l'espace.

Dès lors, l'architecture a permis de donner une interprétation incarnationnelle aux Sept Miséricordes de l'Évangile de Saint Matthieu.

Le premier usage de l'édifice aurait pu être cultuel pour devenir hospitalier (prise en compte du corps et non de l'âme, ce n'est pas une église).

À l'intérieur de la cosmétique théologico-politique de l'incarnation, les hôtels-dieu ont une place à part, en particulier les bourguignons (Cluny, Louhans, Tournus, etc.). On peut, à des fins d'exposition, croiser cette série avec la série basilicale et sa représentation picturale, notamment les ruines antiques en tunnels chez Hubert Robert $^{16}$. À cela s'ajouterait le thème pictural des Sept Miséricordes ou œuvres corporelles (en particulier chez Le Caravage), et pour finir, les salles d'apparat à double niveaux (Caen, Westminster, Montargis, etc.). Dès lors, ce patrimoine classé monument historique devrait apparaître moins fantasmagorique.

\section{BIBLIOGRAPHIE}

Benjamin Walter, «Paris, capitale du XIX siècle », in Écrits français, Paris, Gallimard, coll. « Folio essais ", 1991.

- Sur le concept d'histoire, «thèse 2 », in Écrits français, Paris, Gallimard, coll. « Folio essais », 1991.

- L'CEuvre d'art à l'époque de sa reproductibilité technique, in Écrits français, Paris, Gallimard, coll. « Folio essais », 1991.

Déotte Jean-Louis, « La réception collective d'un film est comme celle d'une architecture urbaine ",_Arts plastiques/cinéma, La Part de l'œil, nº 30, Bruxelles, 2016-2017.

— Le Passage du musée, Paris, L’Harmattan, coll. « Esthétiques », 2017.

- Cosmétiques. Simondon, Panofsky, Lyotard, EMSHA, La Plaine-Saint- Denis, 2018. Web. http:// books.openedition.org/emsha/215

- « L'exposition « Prendre soin » comme déniaisement », Appareil [En ligne], Galerie, mis en ligne le 28 août 2018, consulté le 27 septembre 2018. URL : http://journals.openedition.org/appareil/ 2641 ; DOI : 10.4000/appareil.264

Lacoue-Labarthe Philippe \& Jean-Luc Nancy, L'Absolu littéraire. Théorie de la littérature du romantisme allemand, avec la collaboration d'Anne-Marie Lang, Paris, Le Seuil, 1978.

Payot Daniel, « Coutures et cortèges », Appareil [En ligne], Galerie, mis en ligne le 20 novembre 2017, consulté le 20 juillet 2018. URL : http://journals.openedition.org/appareil/2423

Quincy Quatremère (de), Lettres à Miranda. Sur le déplacement des monuments de l'art de l'Italie (1796), $3^{\mathrm{e}}$ édition augmentée, Introduction par Édouard Pommier, Postface par Emmanuel Alloa, Paris, Éditions Macula, 2017, 1édition 1989. 
- Considérations morales sur la destination des ouvrages de l'art; suivi de Lettres sur l'enlèvement des ouvrages de l'art antique à Athènes et à Rome, Paris, Fayard, coll. « Corpus des œuvres de philosophie en langue française », 1989 [1815], texte revu par Jean-Louis Déotte.

\section{NOTES}

1. In Écrits français, Paris, Gallimard, [1940] 1991.

2. Quincy Quatremère (de), Lettres à Miranda, $3^{\mathrm{e}}$ édition augmentée, Paris, Éditions Macula, 2017 ; Id., Considérations morales sur la destination des ouvrages de l'art, Paris, Éditions Fayard, 1989 [1815].

3. Benjamin Walter, Sur le concept d'histoire, «thèse 2 ", in Écrits français, op. cit., p. 341, 1991.

4. Voir les études de Philippe Lacoue-Labarthe et Jean-Luc Nancy dans L'Absolu littéraire. Théorie de la littérature du romantisme allemand, Paris, Le Seuil, 1979.

5. Payot Daniel, « Coutures et cortèges », Appareil [En ligne], Galerie, mis en ligne le 20 novembre 2017, consulté le 27 septembre 2018. URL : http://journals.openedition.org/appareil/2423 ; DOI : 10.4000/appareil.2423

6. Diane Watteau était la commissaire de cette exposition.

7. Voir entre autres les travaux de Jeanne Susplugas et ceux de Claude Levêque

8. Déotte Jean-Louis, «La réception collective d'un film est comme celle d'une architecture urbaine ", Arts plastiques/cinéma, La Part de l'œil, nº 30, Bruxelles, 2016-2017.

9. Voir le catalogue d'exposition « ...et les marguerites » de Didier Guth. www.didier-guth.com

10. Déotte Jean-Louis, Cosmétiques. Simondon, Panofsky, Lyotard, EMSHA, La Plaine-Saint-Denis, 2018. Web. http://books.openedition.org/emsha/215

11. Walter Benjamin: «Le peintre est à l'opérateur de cinéma ce que le chaman est au chirurgien. Le peintre conserve dans son travail une distance normale vis-à-vis de la réalité de son sujet - par contre le cameraman pénètre profondément les tissus de la réalité donnée. Les images obtenues par l'un et par l'autre résultent de procès absolument différents. L'image du peintre est totale, celle du cameraman faite de fragments multiples coordonnés selon une loi nouvelle. C'est ainsi que, de ces deux modes de représentation de la réalité - la peinture et le film - le dernier est pour l'homme actuel incomparablement le plus significatif, parce qu'il obtient de la réalité un aspect dépouillé de tout appareil - aspect que l'homme est en droit d'attendre de l'œuvre d'art - précisément grâce à une pénétration intensive du réel par les appareils. " in L'Euvre d'art à l'époque de sa reproductibilité technique, in Écrits français, op. cit., 1991, p. 160-161.

12. On pourrait encore développer cette opposition selon le rapport proche/lointain!

13. Château de Caen, XII ${ }^{\mathrm{e}}$ siècle, tête de série pour tout un ensemble de halles, comme l'abbaye de Westminster.

14. Niveau surélevé en raison de l'installation d'un cimetière dans ce qui n'était plus qu'une église.

15. Il faudrait constituer la série de ces salles palatales ou synodales (Sens, Montargis, etc.).

16. Déotte Jean-Louis, Le Passage du musée, Paris, L'Harmattan, coll. « Esthétiques », 2017. 


\section{RÉSUMÉS}

Comment un artefact (un édifice architectural ici) devient-il un objet du patrimoine ou, plus exactement, un objet de patrimoine vivant? L'article avance la notion de prise de site (à distinguer des œuvres in situ), couplée à celles d'idée esthétique et d'analogie, pour penser un rapport à l'œuvre qui ne soit pas soumis aux valeurs de nouveauté.

INDEX

Mots-clés : tradition, exposition, suspension, musée, esthétisation, nouveauté, analogie

\section{AUTEUR}

\section{JEAN-LOUIS DÉOTTE}

Jean-Louis Déotte a été professeur de philosophie à l'Université Paris VIII, Saint-Denis, coordinateur du thème « Esthétique, arts et industries » à la MSH Paris Nord, directeur de la collection « Esthétiques » chez L'Harmattan et enfin, directeur de la revue Appareil. 